\title{
Stromatal Rind Formation in the Brown Rot Fungi
}

\author{
By H. J. WILLETTS \\ Department of Botany, University of Bristol* \\ (Accepted for publication 2 January I968)
}

\begin{abstract}
SUMMARY
The development in culture of a stromatal rind of Sclerotinia fructicola was studied with a light microscope and the surfaces of stromata of the brown rot fungi grown on agar cultures and on fruits were examined with a Stereoscan electron microscope. The hyphae on the surface of some stromata retained their shape, while on other stromata a crust of thickened, collapsed hyphae developed over the outside. In some places rounded cells were seen below the gaps in the crust. The deposit of a melanin-like pigment around and in the walls of exposed stromatal hyphae may possibly increase the resistance of these hyphae to drying and be of importance in survival of the brown rot fungi.
\end{abstract}

Whetzel (1945) attributed the dark colour of the rind cells of sclerotial stromata to impregnated oxidation products of the dead protoplasmic contents. Willetts (I968 $a$ ) suggested that a dark rind forms on all exposed parts of the stromata of the brown rot fungi in contact with air following drying or autolysis of the surface hyphae and that the colour is due to the deposition and accumulation of dark brown melanin-like pigments, around and in the walls of the outer hyphae. Since the latter paper was submitted for publication, the surfaces of young and mature stromata formed in culture and on fruits have been examined with both the ordinary light microscope and the Stereoscan electron microscope. The observations made during the investigation are outlined below.

Sclerotinia fructicola (Wint.) Rehm. was cultured on potato dextrose agar; $S$. fructigena (Aderh. \& Ruhl.), S. laxa (Aderh. \& Ruhl.) and S. laxa forma mali (Wormald) Harrison were grown on malt agar; also apples, pears, cherries, apricots and plums were inoculated with the brown rot species of the genus Sclerotinia found in England. Small pieces (about $6 \times 5 \mathrm{~mm}$ and about I $\mathrm{mm}$. deep) of the stromata that were produced in culture or in the fruits were attached to aluminium stubs with a proprietary adhesive, coated under a high vacuum with a thin layer of gold-palladium alloy and examined in a Stereoscan electron microscope. Similar material sectioned with a freezing microtome was used for light microscope studies.

Plate I, fig. I-4, shows stages in the development in culture of a stromatal rind of Sclerotinia fructicola as seen with a light microscope. The amount of pigmentation increased as the stroma matured and in Pl. I, fig. 4, the outermost surface of the rind is a black layer with two rows of cells beneath. These cells have dark walls and fit closely together to form a pseudoparenchymatous tissue. Enclosed within the rind is a medulla of loosely interwoven hyaline hyphae.

\footnotetext{
* Present address: School of Biological Sciences, University of New South Wales, Australia.
} 
The appearances of the surfaces of old stromata produced in agar cultures when examined with the Steroscan electron microscope are shown in Pl. 2, fig. 5-7. The individual hyphae on the surface of the stroma of Sclerotinia laxa (Pl. 2, fig. 5) retained their shape, even after being subjected to the high vacuum essential for the goldpalladium coating. When young vegetative hyphae and conidiophores were treated in the same way they lost their structure and collapsed. The retention of the shape of the stromatal surface hyphae is probably due to the deposit of the pigment which gives the dark colour to the hyphae. In some areas of the surface of the stromata the hyphae were dried out and formed a crust (Pl. 2, figs. 6, 7), although their walls were thickened. It is probably such areas which, when transversely sectioned, give the black layer shown in Pl. I, fig. 4. The whole of the stroma of S. fructigena (P1. 2, fig. 7) was covered by an incomplete crust of collapsed, thickened hyphae, which may have been a sparse covering of aerial hyphae over the surface of the stroma. Possibly as a result of natural drying, the hyphae had lost their shapes, but had produced a great deal of pigment. Rounded cells can be seen in some of the gaps in the crust and these probably correspond to the outermost pseudoparenchymatous layer of cells beneath the dark outer layer.

The surface of stromata of the brown rot fungi that form inside fruits is similar in appearance to that of stromata that develop in agar cultures. Cherries infected with the fruit-rotting Sclerotinias do not normally develop much aerial mycelium (Willetts, $1968 b$ ) and the rind of the stroma in areas where it ruptures the surface of the cherry is not usually obscured by thin-walled aerial hyphae, since these dry-out and collapse when the stroma matures or when the material is prepared for viewing with the Stereoscan electron microscope. Plate 2, fig. 8, is a Stereoscan electron micrograph of the surface of a cherry infected by Sclerotinia laxa forma mali, showing an exposed surface of the stroma, and Pl. 2, fig. 9, is a higher magnification of one area of this preparation. Numerous hyphae which have retained their shape are seen protruding from the surface. No areas of collapsed hyphae were found in this material.

Stromata are able to survive conditions that are too severe for the ordinary vegetative mycelium, and this is due to the compact structure and chemical composition of the hyphae, particularly those of the outer layers that form the rind. The melanin-like pigment that develops in the hyphae on the surface of the stroma may reduce desiccation of the structure and, under extreme conditions, be of importance in the survival of the fungus. Chet \& Mitchell (1967) found that sclerotial walls of Sclerotium rolfsii Sacc. contain a melanin-like pigment which is absent from hyphal walls and they suggested that the pigment may play some role in the resistance of sclerotia to biological degradation.

Thanks are due to Professor H. E. Hinton, F.R.S., for granting permission to use the Stereoscan electron microscope, which was purchased by the Science Research Council, and to Professor L. E. Hawker for her encouragement and advice. Mr J. W. Heavens operated the instrument and Mr B. Gray assisted in the preparation of the material. 
Journal of General Microbiology, Vol. 52, No. 2

Plate I
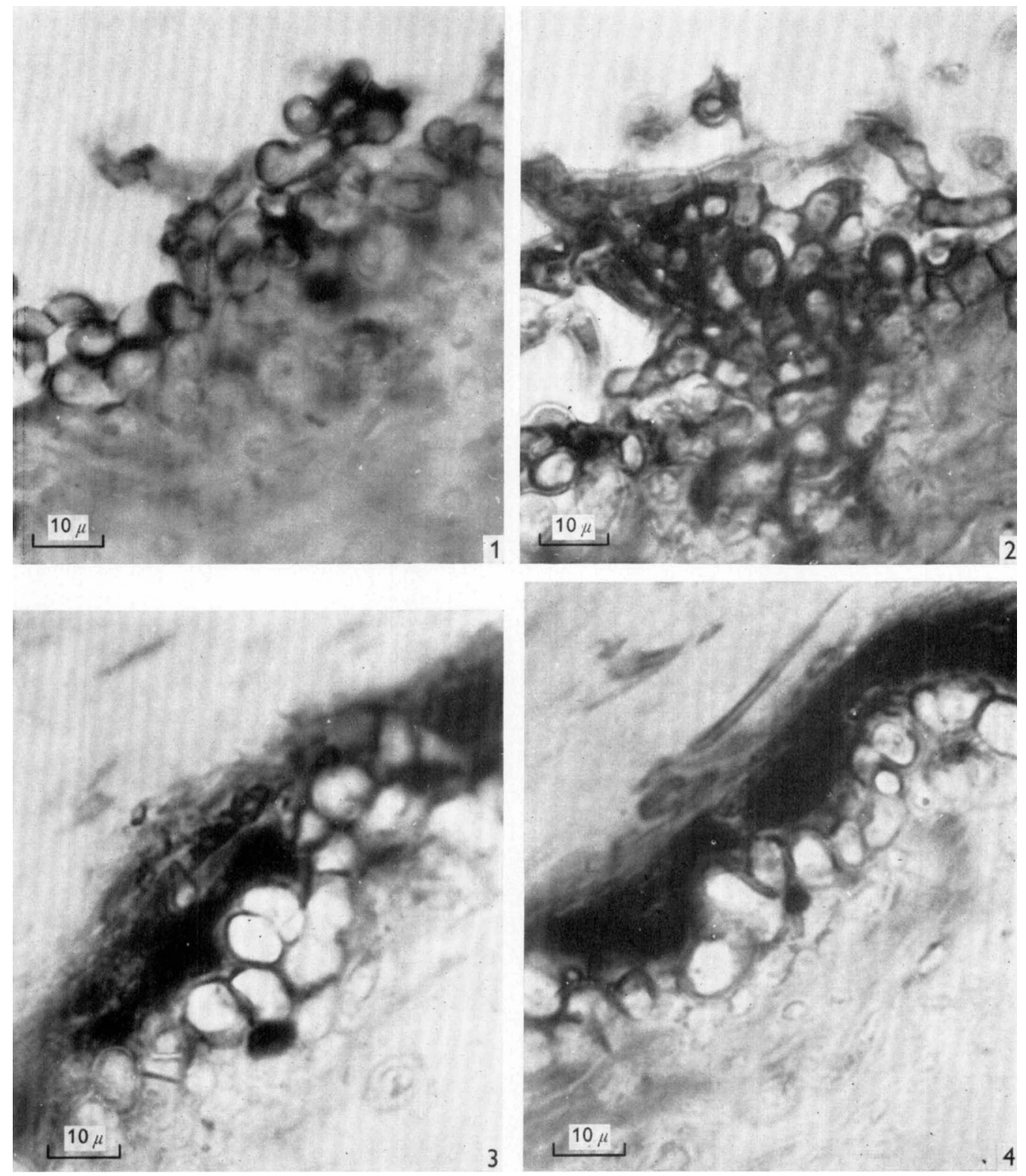

H J. WILLETTS

(Facing p. 272) 

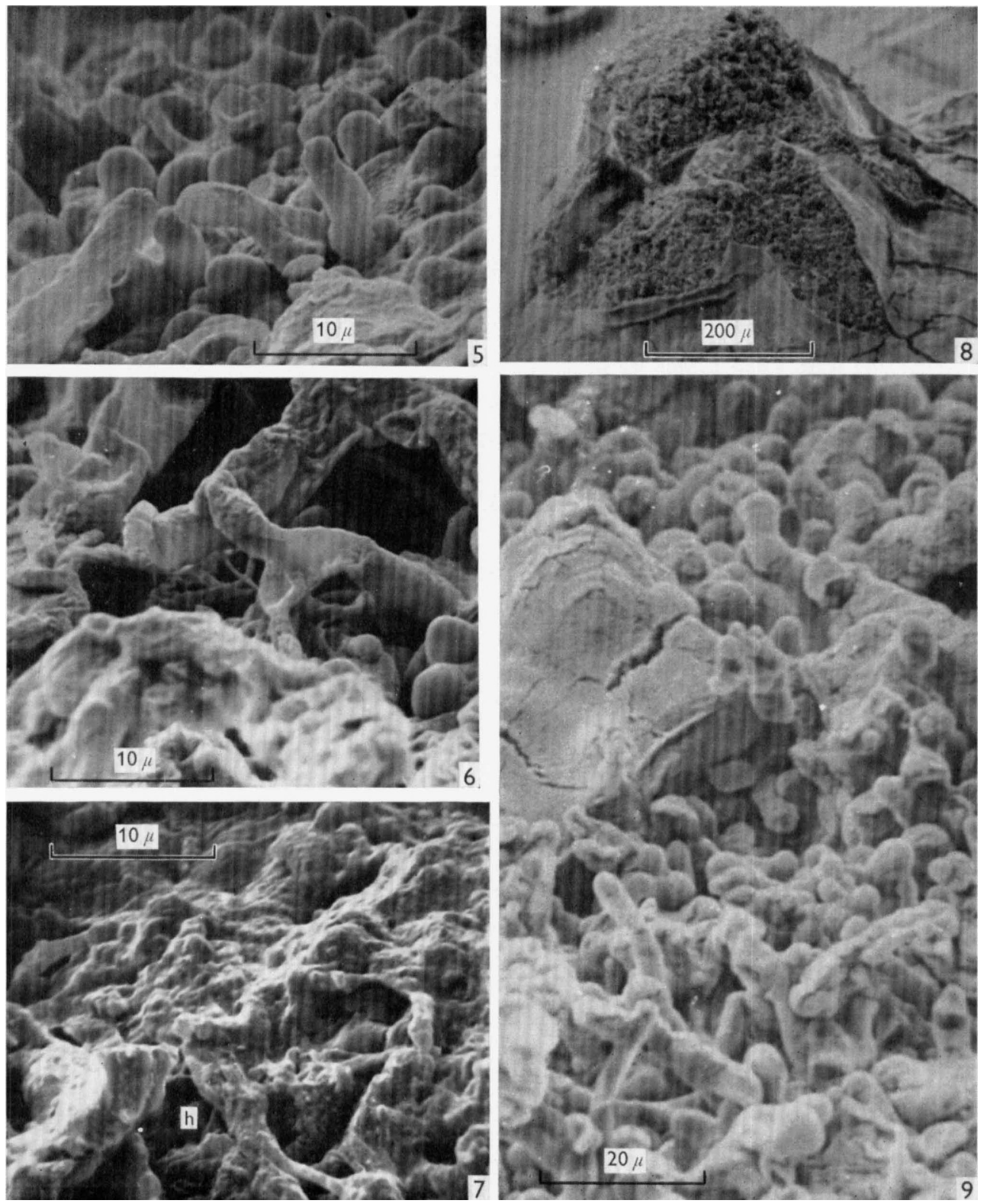

H J. WILLETTS 


\section{REFERENCES}

CHET, Y. H. \& MrtcheLl, R. (1967). Chemical composition of hyphal and sclerotial walls of Sclerotium rolfsii Sacc. Can. J. Microbiol. 13, 137.

WhETZEL, H. H. (1945). A synopsis of the genera and species of the Sclerotiniaeceae, a family of stromatic inoperculate Discomycetes. Mycologia 37, 648.

WILLETTS, H. J. (1968a). The development of stromata of Sclerotinia fructicola and related species. I. In culture. Trans. Br. mycol. Soc. (in the Press.)

WIILETTS, H. J. (I968b). The development of stromata of Sclerotinia fructicola and related species. II. In Fruits. (In preparation.)

\section{EXPLANATION OF PLATES}

Plate I

Figs. $\mathrm{x}-4$. Stages in the development in culture of a stromatal rind of Sclerotinia fructicola as seen with a light microscope. Note the increase in the amount of pigmentation from the least mature (fig. I) to the most mature stroma (fig. 4) and the presence of a dark layer on the outside surface of the latter.

\section{Plate 2}

Stereoscan electron micrographs of the surfaces of mature stromata

Fig. 5. The surface of the stroma of Sclerotinia laxa, showing hyphae that have kept their shapes despite being subject to a high vacuum.

Fig. 6. Another area of the stroma shown in Fig. 5. Note the crust of thickened, collapsed hyphae and the rounded cells in some of the gaps in this crust.

Fig. 7. The surface of a stroma of Sclerotinia fructigena with an extensive crust and several rounded hyphal tips (h), showing through gaps in the crust.

Fig. 8. The surface of a cherry infected by Sclerotinia laxa forma mali, showing an area where the stroma is exposed by a break in the skin of the cherry.

Fig. 9. An area of the same exposed stroma of Sclerotinia laxa forma mali on cherry. Note the absence of a crust and the numerous rounded hyphae protruding from the surface where cherry skin has been ruptured. 